\title{
Neurotrophins Promote Maturation of Developing Neuromuscular Synapses
}

\author{
Ti Wang, Kewei Xie, and Bai Lu \\ Roche Institute of Molecular Biology, Nutley, New Jersey 07110
}

\begin{abstract}
Although the effects of neurotrophins on survival and differentiation of various neuronal populations have been well studied, little is known about their role in synaptic development and function. We have investigated the long-term effects of neurotrophins in the maturation of neuromuscular synapses in Xenopus nerve-muscle cocultures. BDNF and NT-3, but not NGF, elicited significant changes in several properties of spontaneous synaptic currents (SSCs), indicative of more mature synapses. Most synapses treated by the neurotrophins exhibited a bell-shaped distribution of SSC amplitudes, which reflects mature quantal secretion. The neurotrophins also potentiated the efficacy and reliability of stimulus-induced synaptic transmission. Moreover, BDNF and NT-3 increased the levels of the synaptic vesicle proteins, synaptophysin, and synapsin $I$ in the spinal neurons. The number of varicosities per neuron also showed a significant increase after neurotrophin treatment. The effects of the neurotrophins appear to be mediated by the Trk family of receptor tyrosine kinases, primarily through a presynaptic mechanism. These results suggest that BDNF and NT-3 promote functional maturation of synapses.
\end{abstract}

[Key words: neurotrophins, synapse maturation, neuromuscular junction, nerve-muscle coculture, spontaneous and evoked synaptic currents, synaptic vesicle proteins]

Synapse formation is a well-concerted event that involves multiple stages. After initial synaptic contact is made, the synapse undergnes a series of maturation processes (for a recent review, see Hall and Sanes, 1993). Previous studies using Xenopus nerve-muscle culture have provided a wealth of information on the morphological and physiological events associated with the maturation of neuromuscular synapses. These events include a gradual increase of the frequency and amplitude as well as a decrease in the rise time of spontaneous synaptic currents (SSCs). The most characteristic feature of maturation is the transition from a skew to a bell-shaped distribution of SSC amplitudes. Moreover, the amplitudes of impulse-evoked synaptic currents (ESCs) become much larger and more consistent (Kidokoro et al., 1980; Kidokoro, 1984; Evers et al., 1989; Lu et al., 1992). The enhancement of synaptic efficacy is accompanied by

\section{Received Dec. 5, 1994; revised Feb. 14, 1995; accepted Feb. 17, 1995}

The neurotrophins were kindly provided by Genentech, Inc., South San Francisco, CA. We express gratitude to Drs. Mu-ming Poo, J. Connor, and S. Udenfriend for helpful discussions and critical comments of the manuscript, Dr. Wen-mei $\mathrm{Fu}$ for advice on some of the experiments, and Dr. F. Valtorta for anti-synaptophysin antibody.

Correspondence should be addressed to Bai Lu, Ph.D. at the above address. Copyright $(C) 1995$ Society for Neuroscience 0270-6474/95/154796-10\$05.00/0 changes in the structure of the synapses. Thus, the nerve terminals exhibit clusters of synaptic vesicles; both pre- and postsynaptic membranes thicken; basal lamina material appears in the synaptic cleft; and acetylcholine $(\Lambda \mathrm{Ch})$ receptors start to aggregate on the postsynaptic membrane (Kelly and Zachs, 1969; Kullberg et al., 1977; Nakajima et al., 1980; Takahashi et al., 1987; Buchanan et al., 1989).

Although the molecular mechanism underlying synaptic maturation remains unknown, interaction between pre- and postsynaptic elements appears to be crucial. At the presynaptic site, vesicle-associated phosphoprotein synapsin I has been suggested to participate in the maturation process (Lu et al., 1992; Valtorta et al., 1995). On the other hand, extensive studies of synaptic development have long suggested that postsynaptic muscle cells provide soluble factors that retrogradely regulate various properties of motor neurons, including the maturation of neuromuscular synapses (Purves and Lichtman, 1985; Oppenheim, 1991; Yin and Oppenheim, 1992; Hall and Sanes, 1993). Neurotrophins may be a potential class of the factors that serve such a role.

Neurotrophins are a family of proteins that includes nerve growth factor (NGF), brain-derived neurotrophic factor (BDNF), neurotrophin-3 (NT-3), and neurotrophin-4/5 (NT-4/5). Extensive studies in the past few years have elucidated the important roles that the neurotrophins play in neuronal survival and differentiation in both the PNS and CNS (Barde, 1989; Thoenen, 1991). The functions of neurotrophins on distinct neuronal populations are mediated by the cellular expression of the Trk family of tyrosine kinase receptors (Chao, 1992; Barbacid, 1993). Trk is predominantly activated by NGF, Trk B by BDNF, NT-3, and NT-4/5, and Trk C by NT-3. Recently, neurotrophins, particularly BDNF, have been shown to reduce the death of motor neurons in the spinal cord both during development and after axotomy in the adult (Oppenheim et al., 1992; Sendtner et al., 1992; Yan et al., 1992; Henderson et al., 1993; Koliatsos et al., 1993). Trk B and trk C mRNAs are expressed in motor neurons (Henderson et al., 1993; Ip et al., 1993; Koliatsos et al., 1993; Wong et al., 1993), while BDNF and NT-3 are in muscle cells (Schecterson and Bothwell, 1992; Henderson et al., 1993; Koliatsos et al., 1993). Although BDNF and NT-3 knockout mice do not exhibit obvious motor neuron deficits (Ernfors et al., 1994a,b; Farinas et al., 1994; Jones et al., 1994), genetic deletion of trk $B$ receptor results in a severe reduction of the number of motor neurons in the spinal cord (Klein et al., 1993). Thus, BDNF and NT-3 may have compensatory role during motor neuron development. Taken together, these results support a model in which neurotrophins serve as muscle-derived target factors that promote motor neuron survival. 
Do neurotrophins also regulate the development of neuromuscular synapses? Recent work by Lohof et al. indicate that synaptic activities are rapidly potentiated by acute exposure to BDNF and NT-3 (Lohof et al., 1993). However, during normal development in vivo, synapses are probably exposed to limited but constant amount of neurotrophins. In the present study, we investigated the chronic effects of neurotrophins on developing neuromuscular synapses in Xenopus nerve-muscle cultures. We now provide evidence that BDNF and NT-3, but not NGF, elicit both electrophysiological and morphological changes that reflect synaptic maturation. These observations raise the possibility that members of the neurotrophin gene family serve as retrograde signals to promote the maturation of neuromuscular synapses.

\section{Materials and Methods}

Culture preparation. Xenopus nerve-muscle cultures were prepared according to the procedure described previously (Tabti and Poo, 1991; Lu et al., 1992). Briefly, the neural tube and the associated myotomal tissue of 1-d-old Xenopus embryos (stage 20 to 22 according to Nieuwkoop and Faber, 1967) were dissociated in $\mathrm{Ca}^{2+} / \mathrm{Mg}^{2+}$-free saline supplemented with EDTA $(58.2 \mathrm{~mm} \mathrm{NaCl}, 0.7 \mathrm{~mm} \mathrm{KCl}, 0.3 \mathrm{~mm}$ EDTA, pH 7.4) for 15-20 min. The cells were plated on clean glass coverslips. The cultures were grown for $2-3 \mathrm{~d}$ at room temperature $\left(20-22^{\circ} \mathrm{C}\right)$ before used for experiments. The culture medium consisted ( $\mathrm{vol} / \mathrm{vol})$ of $50 \%$ Leibovitz L-15 medium (Sigma), $1 \%$ fetal calf serum (GIBCO), and $49 \%$ Ringer's solution (115 mM NaCl, $2 \mathrm{~mm} \mathrm{CaCl}_{2}, 2.5 \mathrm{~mm} \mathrm{KCl}$ $10 \mathrm{~mm}$ HEPES, $\mathrm{pH}$ 7.6). Various neurotrophins $\left(5 \times 10^{-10} \mathrm{M}\right.$, NGF, BDNF, NT-3, kindly provided by Genentech, Inc., South San Francisco, CA) were added to the cultures $6 \mathrm{hr}$ after plating, when cells were completely settled. In some experiments, specific inhibitor for trk tyrosine kinase k252a $(0.2 \mu \mathrm{M}$, Biomol Res. Lab., Inc., Plymouth Mccting, PA) was added together with NT-3 to block the neurotrophin action.

Electrophysiology. Synaptic currents were recorded from innervated muscle cells by whole-cell recording methods (Hamill et al., 1981; Lu et al., 1992), at room temperature in culture medium. The solution inside the whole-cell recording pipette contained $150 \mathrm{mM} \mathrm{KCl}, 1 \mathrm{~mm}$ $\mathrm{NaCl}, 1 \mathrm{mM} \mathrm{MgCl}$, and $10 \mathrm{mM}$ HEPES buffer ( $\mathrm{pH}$ 7.2). To elicit evoked synaptic currents, square current pulses $(0.5 \mathrm{msec}, 0.5-5 \mathrm{~V}, 0.05$ $\mathrm{Hz}$ ) were applied to presynaptic neurons at soma with a glass microelectrode filled with Ringer's solution. All data were collected by a patch-clamp amplifier (EPC-7), with a current signal filter at $3 \mathrm{kHz}$. The data were stored on a videotape recorder for later playback on a storage oscilloscope (Textronic TDS 420) and a chart recorder (Gould EasyGraf 240), or analysis by a microcomputer. The amplitude, rise and decay times of SSCs and ESCS werc analyzcd by SCAN program (Dagan, Inc.).

Cell manipulation experiment. Spherical myocytes (myoballs) from I d control cultures were used as a detecting probe to measure $\mathrm{ACh}$ release from neurons in 3-d-old cultures treated with or without NT-3 Two coverslips from $3 \mathrm{~d}$ cultures, one treated with and the other without NT-3, were brought together to a third coverslip of 1-d-old culture. An isolated myoball on the $1 \mathrm{~d}$ culture coverslip was first whole-cell clamped at resting membrane potential by patch-clamping method. The cell was then detached from the culture substratum with the recording pipette and repositioned on top of free nerve terminals of 3-d-old neurons in either the control or NT-3-treated coverslip. These isolated terminals were never in contact with muscle cells before, although other branches of the same spinal neuron may do so. The same myoball was used to probe many terminals and the synaptic currents of control and NT-3-treated neurons were compared in the same dish.

Immunocytochemistry and image processing. Xenopus nerve-muscle cocultures, $2 \mathrm{~d}$ and $3 \mathrm{~d}$ old, were fixed with $4 \%$ paraformaldehyde in $40 \mathrm{~mm} \mathrm{NaCl}, 10 \mathrm{mM} \mathrm{MgCl}, 10 \mathrm{~mm}$ EGTA, and $20 \mathrm{~mm}$ HEPES, pH 7.4. Immunofluorescent staining was performed through the following steps: (1) washing $1 \times$ in phosphate-buffered saline (PBS), $\mathrm{pH} 7.4 ; 1 \times$ with PBS containing $0.2 \%$ Triton $\mathrm{X}-100$, and then $2 \times$ with PBS; $(2)$ incubation in $0.1 \mathrm{M}$ glycine $/ \mathrm{NaOH}, \mathrm{pH} 7.4$ for $15 \mathrm{~min}$; (3) incubation in blocking solution ( $3 \%$ BSA in PBS) for $1 \mathrm{hr}$; (4) incubation with primary antibodies against Xenopus synaptophysin (Valtorta et al., 1988), and Xenopus synapsin I (I u et al., unpublished observations), diluted 1:200 in blocking solution; (5) wash with blocking solution twice; (6) incubation with FITC conjugated goat anti-rabbit IgG anti- body (1:200) in blocking solution; (7) wash, first with blocking solution, then with PBS, and finally with water; (8) the coverslips were mounted onto glass slides with Vectorshield mounting solution (Vector) and examined with a Zeiss microscope. We used Adobe PHOTOSHOP program for semiquantitation of immunocytochemistry. Images of immunofluorescence staining were photographed with the same exposure time (30 $\mathrm{sec}$ ) and scanned into the program. All varicosities along the axon of a spinal neuron (minimum of 10 ) were measured by the program to obtain a median value of fluorescence intensity of that particular neuron. The median value was subtracted by the average nonspecific fluorescence from the same image. The data from five to eight spinal neurons in each treatment condition were then averaged, and divided by that of control. The averaged values from all treatment conditions were presented as relative fluorescence intensities and subject to Student's $t$ test.

\section{Results}

Effects of neurotrophins on spontaneous synaptic currents

Developing neuromuscular synapses were studied in Xenopus nerve-muscle cultures grown in the absence or presence of various neurotrophins. In control cultures, synaptic contacts are established within the first day after plating, and synaptic activity undergoes a maturation process that takes 4-5 d (Kidokoro et al., 1980; Kidokoro, 1984; Evers et al., 1989; Lu et al., 1992). We chose to study synapses during the transition period, in the 2-d- and 3-d-old cultures. Figure 1 shows the effects of NT-3 on spontaneous synaptic currents (SSCs) recorded from 2-d-old cultures. Chronic treatment of NT-3 $\left(5 \times 10^{-10} \mathrm{M}\right.$, in all experiments) dramatically potentiated the spontaneous synaptic activity. The mean amplitude and frequency of the SSCs recorded from NT-3-treated synapses in $2 \mathrm{~d}$ cultures were 2 times and 2.5 times those of control cultures (Fig. 1A, Table 1). Similar effects of NT-3 were observed for 3-d-old cultures (Table 1).

An important measurement of synaptic maturation is the amplitude distribution of SSCs. The distribution from an immature synapse is typically skewed towards small amplitudes and can be fitted by an exponential curve. During the process of maturation, the distribution becomes biphasic. In addition to the exponential component observed in immature synapses, many higher amplitude events clustered to form a "bell" reminiscent of normal distribution observed in adult synapses (Kidokoro, 1984; Lu et al., 1992). The immature exponential distribution is regarded as "skcwed," while the maturing, biphasic distribution as "bell-shaped." This characteristic bell-shaped distribution of SSC amplitudes reflects the development of a well-defined ACh quantal unit and therefore can be used as an electrophysiological measure of synaptic maturation. Treatment with NT-3 resulted in a transition from skew to bell-shaped SSC amplitude distributions (Figs. 1, 2). Representative histograms for 2-d-old synapses treated with or without NT-3 are shown in Figure $1 B$. A more dramatic effect on SSC distribution was observed in $3 \mathrm{~d}$ cultures treated with NT-3, although the peaks varied among synapses (Fig. 2B). A bell-shaped distribution appeared at high SSC amplitudes for many $2 \mathrm{~d}$ synapses (63\%) and most of $3 \mathrm{~d}$ synapses $(80 \%)$ in NT-3-treated cultures. In contrast, more than $80 \%$ of control synapses in $2 \mathrm{~d}$ and $3 \mathrm{~d}$ cultures exhibited a skewed SSC amplitude distribution.

Another parameter of synaptic maturation is the time course of SSCs. At an immature neuromuscular synapse, the area of contact is usually small and irregular in shape, and the distances between transmitter release sites and the postsynaptic membrane are large and variable (Buchanan et al., 1989). This morphological feature leads to synaptic currents with a slow and variable rise time (Evers et al., 1989). However, when neurons were grown in NT-3 for $2 \mathrm{~d}$, the SSCs exhibited a faster and more 


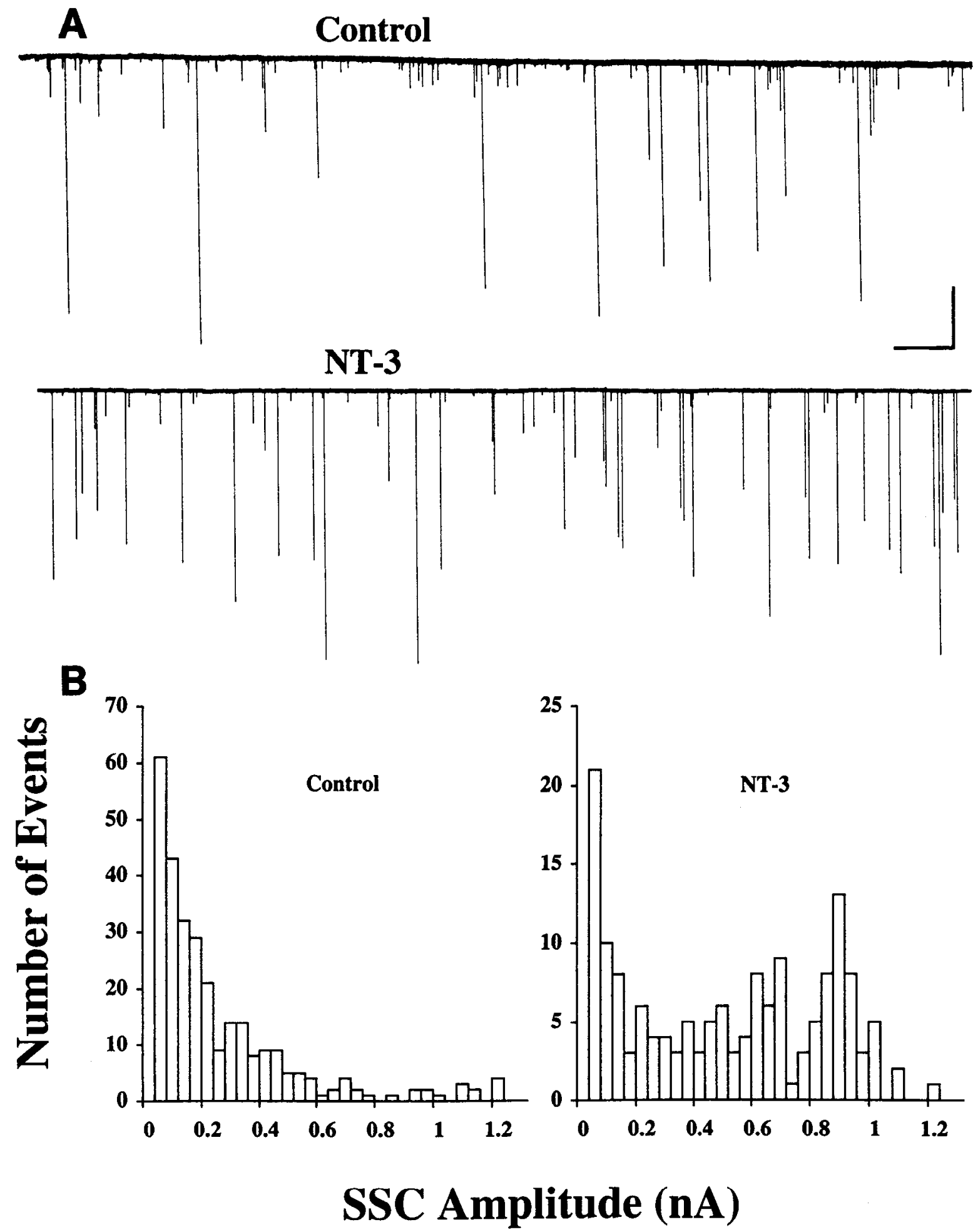

Figure 1. Effect of NT-3 on spontaneous synaptic activity in 2-d-old cultures. A, Membrane currents recorded from innervated myocytes from control (upper) or NT-3-treated (lower) cultures. Spontaneous synaptic currents (SSCS) of varying amplitudes are shown as downward deflections $\left(V_{n}=-70 \mathrm{mV}\right.$, filtered at $150 \mathrm{~Hz}$ ). Note the increase in frequency and mean amplitude of the SSCs at the NT-3-treated synapse. Calibration: 0.5 nA, $40 \mathrm{sec}$. B. Histograms of SSC amplitude distribution constructed from recordings of control and NT-3-treated synapses shown in $A$.

consistent rise time compared with those of controls (Table 1). The average rise time for NT-3-treated $2 \mathrm{~d}$ and $3 \mathrm{~d}$ synapses were $29 \%$ and $32 \%$ shorter than for controls. The rise time in 3-d-old cultures also become less variable after NT-3 treatment (Table 1). We used the average coefficient of variation $(\mathrm{CV}=$
$\mathrm{SD} /$ mean) of SSC rise time to measure this tendency. There were statistically significant decreases for $\mathrm{CV}$ of $\mathrm{SSC}$ rise time in 3 $\mathrm{d}$, but not $2 \mathrm{~d}$ synapses cultured in NT-3, suggesting that longer treatment with NT-3 is needed for the appearance of morc consistent SSC rise time (Table 1). Interestingly, NT-3 did not affect 
Table 1. Effects of neurotrophins on spontaneous synaptic currents

\begin{tabular}{|c|c|c|c|c|c|c|c|}
\hline Conditions & $\begin{array}{l}\text { Rise time } \\
\text { (Insec) }\end{array}$ & $\begin{array}{l}\text { Rise time CV } \\
(\%)\end{array}$ & $\begin{array}{l}\text { Decay time } \\
\text { (misec) }\end{array}$ & $\begin{array}{l}\text { Amplitude } \\
(\mathrm{pA})\end{array}$ & $\begin{array}{l}\text { Amplitude CV } \\
(\%)\end{array}$ & $\begin{array}{l}\text { Frequency } \\
\text { (events/IIIII) }\end{array}$ & $n$ \\
\hline $2 \mathrm{~d}$ Control & $1.3 \pm 0.1$ & $5.0 \pm 0.4$ & $10.2 \pm 0.9$ & $238 \pm 23$ & $8.27 \pm 1.1$ & $4.1 \pm 0.7$ & 11 \\
\hline NT-3 & $0.9 \pm 0.2^{*}$ & $5.7 \pm 2.0$ & $10.1 \pm 1.0$ & $488 \pm 119^{* * *}$ & $8.54 \pm 1.8$ & $10.5 \pm 3.9 *$ & 6 \\
\hline NT-3 & $0.9 \pm 0.1 *$ & $1.9 \pm 0.2^{* *}$ & $10.7 \pm 1.4$ & $364 \pm 68^{*}$ & $3.7 \pm 0.3^{* *}$ & $17.8 \pm 2.5^{* *}$ & 8 \\
\hline BDNF & $1.0 \pm 0.1^{*}$ & $5.8 \pm 1.0$ & $10.3 \pm 0.8$ & $403 \pm 49 * *$ & $7.7 \pm 0.7$ & $12.9 \pm 1.5^{*}$ & 12 \\
\hline NGF & $1.4 \pm 0.1$ & $5.0 \pm 0.7$ & $11.0 \pm 1.4$ & $272 \pm 36$ & $7.7 \pm 0.8$ & $6.7 \pm 1.8$ & 8 \\
\hline
\end{tabular}

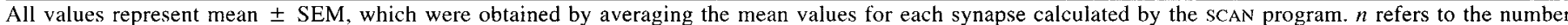
of synapses recorded. $\mathrm{CV}=\mathrm{SD} / \mathrm{mean}$. The data were subject to Student's $t$ test.

$* p<0.05$.

$* * p<0.01$.

A
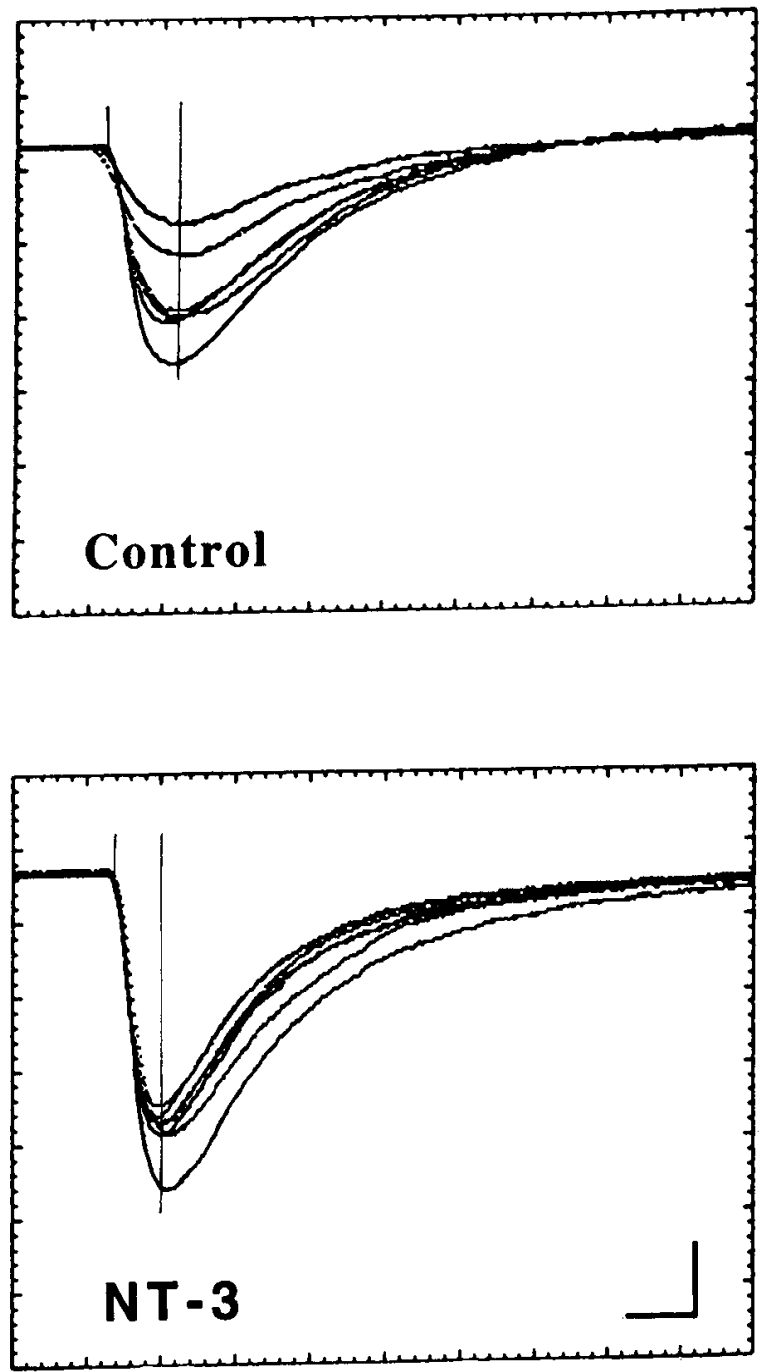
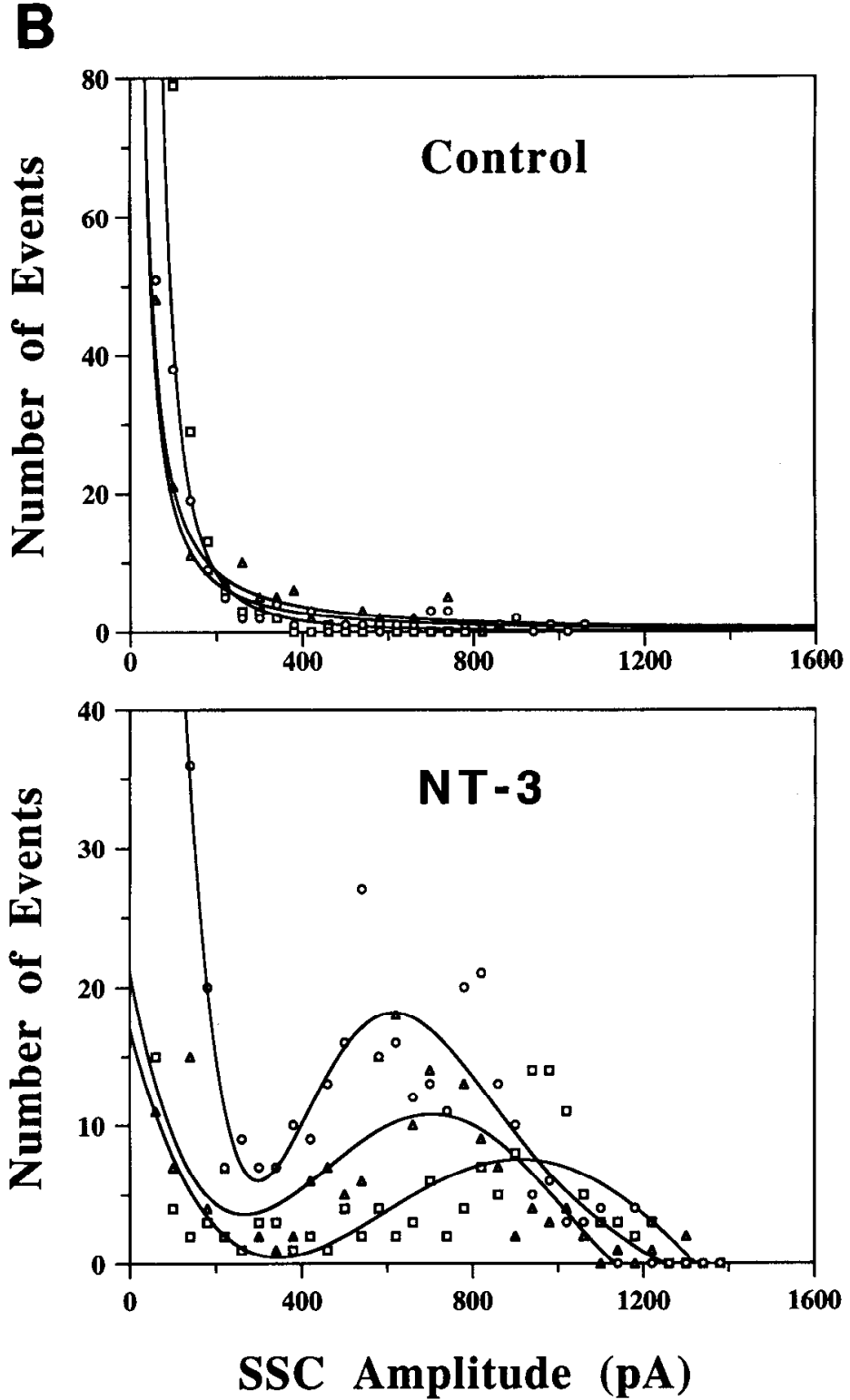

Figure 2. Effect of NT-3 on spontaneous synaptic activity in 3-d-old cultures. A, Superimposed oscilloscopic traces of SSCs at higher time resolution (filtered at $2.5 \mathrm{kHz}$ ). Note the decrease in the rise time of SSCs after NT-3 treatment. Calibration: $0.5 \mathrm{nA}, 1 \mathrm{msec} . B$, Composite amplitude distributions of SSCs. Three representatives from either control or NT-3-treated synapses are shown. The control data have becn fitted by exponential curves, and the NT-3-treated data fitted by polynomial curves to show peaks of the bell-shaped distribution of SSC amplitude. 


\section{Control}

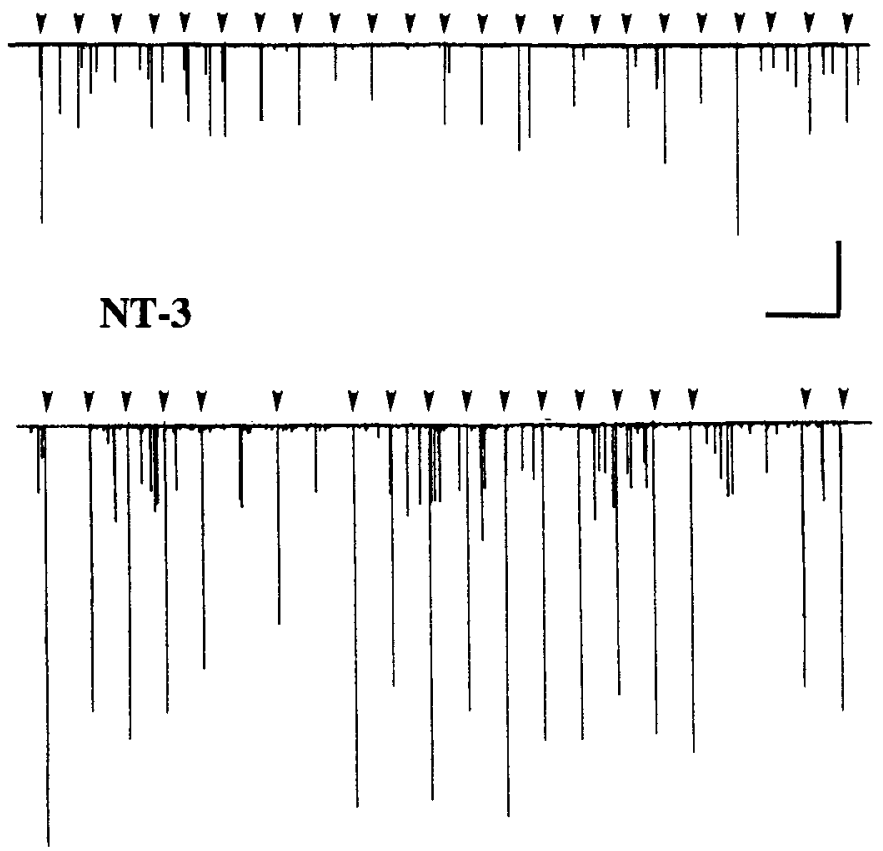

Figure 3. Effect of NT-3 on impulse-evoked synaptic activity. The continuous traces represent membrane currents recorded from innervated myocytes in 2-d-old cultures treated with (lower) and without (upper) NT-3. The presynaptic neuron was stimulated at the soma extracellularly $(0.5-5 \mathrm{~V}, 0.1 \mathrm{msec}$ duration, $0.05 \mathrm{~Hz})$ over the duration of the recording to initiate action potentials. Evoked synaptic currents (ESCs) appear as large, regularly spaced downward deflections marked by arrowheads in the current traces amidst randomly occurring SSCs $\left(V_{h}=-70 \mathrm{mV}\right.$, filtered at $\left.150 \mathrm{~Hz}\right)$. Calibration: $2 \mathrm{nA}, 0.5 \mathrm{~min}$.

the decay time of SSCs, which reflects the open time of postsynaptic $\mathrm{ACh}$ receptors. These findings raise the possibility that NT-3 promotes the formation of a more mature synaptic junction with a closer and even pre- and post synaptic membrane apposition.

The idea that NT-3 induces structural changes in neuromuscular synapses is further supported by the fact that the potentiation of SSCs persisted even after withdrawal of NT-3 from 3 d cultures. In four experiments, SSCs were recorded from the same synapses first in NT-3 containing culture media. The cultures were then thoroughly washed and replaced with Ringer solution. There was no statistical difference in the amplitude and frequency of SSCs recorded hefore and after replacement of NT-3 media. The electrophysiological parameters of the SSCs recorded in the Ringer replacement media appeared still much more mature than those in control cultures.

The activity of NT-3 is known to be mediated by trk receptor tyrosine kinases (Chao, 1992; Barbacid, 1993). The membrane permeable drug $\mathrm{k} 252 \mathrm{a}$, at a concentration of 50-200 nM, specifically inhibits autophosphorylation and activation of the Trk family of receptors without affecting other tyrosine kinases (Berg et al., 1992; Nye et al., 1992; Tapley et al., 1992). Therefore, it has been frequently used to selectively block the biological action of neurotrophins. The effect of NT-3 on synaptic maturation was substantially inhibited when cultures were grown in NT-3 together with $\mathrm{k} 252 \mathrm{a}$. Virtually all the changes in electrophysiological parametcrs (cxccpt SSC amplitude) induced by NT-3 were reversed by $\mathrm{k} 252 \mathrm{a}$ (Table 1 ). The differences in av-

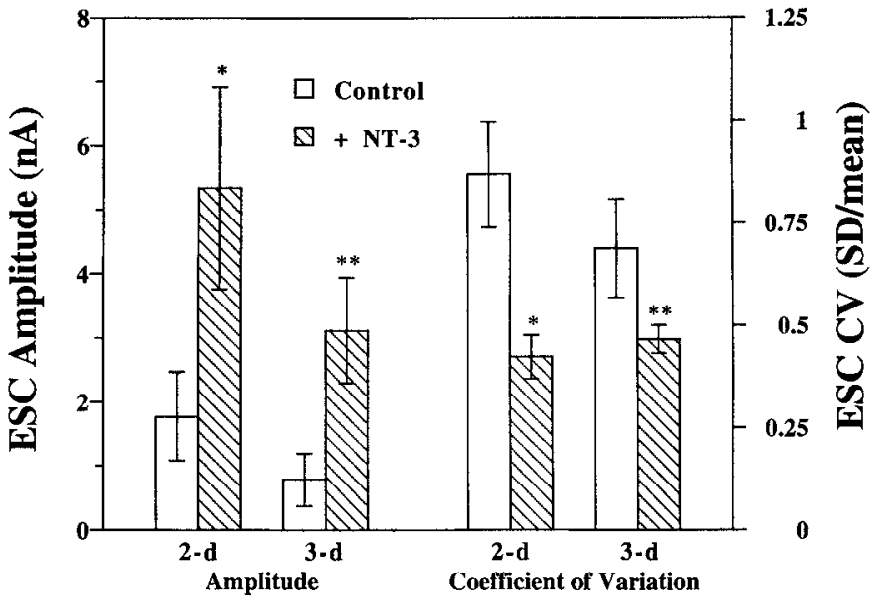

Figure 4. Quantitative comparison of NT-3 effects on ESC amplitudes and variations in $2 \mathrm{~d}$ and $3 \mathrm{~d}$ cultures. Approximately 20 to 50 evoked currents were recorded from each individual synapse. The mean amplitudes and coefficient of variation (CV, defined as SD/mean of ESC amplitude) of ESCs were calculated individually for each group of synapses before averaging. Number of synapses used in each group: seven control and nine NT-3-treated synapses in $2 \mathrm{~d}$ cultures; and six control and seven NT-3-treated synapses in $3 \mathrm{~d}$ cultures. Error bars are standard errors. The data were subjected to Student's $t$ test. ${ }^{*} p<0.05 ;{ }^{*} p<<$ 0.01

erage SSC amplitudes between control and NT-3/k252a were not statistically significant. Thus, activation of trk receptor tyrosine kinases by endogenous NT-3 may be an important step in strengthening the neuromuscular synapses during development.

To determine whether the NT-3 action was specific, we examined the effects of other neurotrophins on the properties of SSCs. NGF $\left(5 \times 10^{-10} \mathrm{M}\right)$ had no effect on any of the parameters analyzed (Table 1$)$. In contrast, BDNF $\left(5 \times 10^{-10} \mathrm{M}\right)$ potentiated spontaneous synaptic activity, although its effects were less dramatic than those of NT-3 (Table 1). In 3-d-old cultures treated with BDNF, the amplitudes and frequency of SSCs increased $68 \%$ and $107 \%$ over controls, respectively. The rise and decay times of SSCs did not change. There were no statistically significant changes in the variability of amplitude and rise time (as reflected by their coefficients of variation). The differential effects of neurotrophins indicate not only the specificity, but also the relative potency of various neurotrophins in regulating neuromuscular synapses.

\section{Effects of NT-3 on evoked synaptic currents}

Functional synaptic transmission is measured by the impulseevoked synaptic response. We therefore compared the evoked synaptic currents (ESCs) of neuromuscular synapses grown in the absence or presence of NT-3. In these studies suprathreshold stimuli were applied to the soma of the neurons to fire action potentials at a low frequency $(0.05 \mathrm{~Hz})$ and the ESCs were recorded from the postsynaptic muscle cells. Figure 3 shows the effects of NT-3 on (ESCs) recorded from 2-d-old cultures. Chronic treatment of spinal neurons with NT-3 resulted in a three- and fourfold increase in ESC amplitude in 2-d- and 3-dold cultures, respectively (Figs. 3, 4). Moreover, the variation of the amplitudes became much smaller, indicating that more mature and reliable synaptic functions were established. The average CV of ESC amplitudes of NT-3-treated neurons in 2-d- and 3 -d-old cultures was significantly smaller than those of control neurons (Fig. 4). The changes in evoked synaptic activity indi- 
cate that NT-3 enhances the efficacy and reliability of functional synaptic transmission at developing neuromuscular synapses.

\section{Enhancement of presynaptic ACh secretion by NT-3}

Changes in synaptic activity could be due to pre- or postsynaptic mechanisms. To test directly whether NT-3 induces an increase in neurotransmitter release we used a cell manipulation technique to detect transmitter secretion in isolated, frec presynaptic nerve terminals (Xie and Poo, 1986). A 1-d-old spherical myocyte (myoball) was whole-cell clamped and detached from culture substratum with the patch pipette. The same myoball was then manipulated into contact with the free nerve terminals of either control or NT-3-treated neurons in 3-d-old cultures. These free terminals were never in contact with any myocytes before, and their ability to release $\mathrm{ACh}$ thus reflects faithfully the presynaptic property. SSCs were then recorded from each terminal for about $10-20 \mathrm{~min}$ using the whole-cell recording pipette attached to the myoball. The mean amplitude and frequency of SSCs in control terminals were $123 \pm 20 \mathrm{pA}$ and $4.5 \pm 1.2$ events/min, whereas those of NT-3-treated terminals were 345 $\pm 65 \mathrm{pA}$ and $11.3 \pm 2.8$ events/min, respectively ( $n=6$ pairs). The values from the NT-3-treated group were significantly higher than those from the control group ( $t$ test, $p<0.01$ ). Interestingly, $66 \%$ of NT-3-treated terminals even exhibited bell-shape like distributions of SSC amplitudes (data not shown). Thus, it appears that NT-3 produces significant presynaptic effects independent of postsynaptic cells.

\section{Morphological changes induced by neurotrophins}

To examine the morphological changes induced by the neurotrophins, we measured the levels of synaptophysin and synapsin I, two synapse-specific proteins. Since there were only limited a number of neurons in the nerve-muscle cultures, it was difficult to measure the levels of these proteins, even with the most sensitive immunoblotting methods. Instead, immunocytochem istry was used to detect changes in the levels and distribution of these proteins in the spinal neurons. Synaptophysin, an integral membrane protein of the synaptic vesicle (Sudhof and Jahn, 1991), has been shown to be required for functional synaptic transmission (Alder et al., 1992). We were able to detect synaptophysin in immature neurons on the first day of culture, indicating that its expression is associated with early neural development. In 2 -d-old control cultures, the protein was evenly distributed throughout the cell body and neurites of spinal nelrons. Treatment with BDNF and NT-3 for $2 \mathrm{~d}$ caused a marked segregation of synaptophysin into varicosities (enlargements along the axons) along the axons and nerve terminals (data not shown). The staining of synaptophysin became gradually segregated in 3-d-old culture (Fig. $5 A$ ), but the segregation was more pronounced in BDNF- or NT-3-treated cultures (Fig. $5 C, D$ ). The staining also looked much brighter and appeared to be concentrated on varicosities in the BDNF- and NT-3-treated cultures (compare Fig. $5 A, C, D$ ). We used an imaging analysis program for semiquantitation of the staining. The staining intensities from all varicosities along the axon of a spinal neuron (minimum of 10) were subtracted from nonspecific fluorescence from the same image and averaged. In BDNF- and NT-3-treated neurons, the intensity of the staining on the varicosities increased $36 \%$ and $41 \%$ (Fig. 7). NGF had no effect on the distribution and intensity of synaptophysin (Figs. $5 B, 7$ ). The effects of NT-3 were virtually prevented by cotreatment with $\mathrm{k} 252 \mathrm{a}$, suggesting that the neurotrophin effects are mediated by trk receptor tyrosine kinases (Figs. 5E, 7).

We also investigated the effects of NT-3 on the staining of synapsin I, a neuronal phosphoprotein associated with synaptic vesicles (Greengard et al., 1993). Several lines of evidence suggest that synapsin I may be one of the molecules involved in the maturation of neuromuscular synapses ( $\mathrm{Lu}$ et al., 1992; Valtorta et al., 1995). Using an antibody specific for Xenopus synapsin I, we found that the level of synapsin I was low in the immature neurons in 2-d- and 3-d-old control cultures (Fig. 6). Unlike synaptophysin, synapsin I immunoreactivity was usually associated with nerve terminals and varicosities where synaptic vesicles are concentrated. There were a few synapsin I positive spots on the neurites of spinal neurons in $2 \mathrm{~d}$ cultures (not shown), and the number of spots increased slightly in $3 \mathrm{~d}$ cultures (Fig. 6A). Most of the synapsin I positive neurites were in contact with myocytes, raising the possibility that endogenous NT-3 released from target myocytes induces synapsin I expression in innervating neurons. In contrast to controls, the level of the staining increased significantly in neurons cultured in BDNГ or NT-3 for $3 \mathrm{~d}$ (Fig. 6C,D). Computer imaging analysis showed that the intensity of synapsin I staining increased by $75 \%$ and $102 \%$ after BDNF and NT-3 treatments (Fig. 7). Virtually all nerve terminals and varicosities exhibited bright synapsin I positive staining. Again, NGF did not have any effect on synapsin I expression, and k252a blocked the NT-3 effect (Fig. 6B,E).

In addition to enhancing the levels of synapsin I and synaptophysin staining, BDNF and NT-3 also elicited more varicosities per spinal neuron. Varicosities were defined as synaptophysin and synapsin I positive enlargement along the neurites of spinal neurons. Because of the heterogeneity of the number of varicosities per unit length of neurite, we counted the total number of varicosities per neuron. As shown in Figures 5 and 6 , significantly more varicosities were found in neurons grown in BDNF and NT-3 than those in control cultures. Quantitative measurements indicated that the total number of varicosities per neuron increased $104 \%$ and $152 \%$ in $3 \mathrm{~d}$ cultures treated with BDNF and NT-3 compared with control and NGF-treated cultures (Fig. 8). k252a also blocked the effect of NT-3 on the number of varicosities. These results suggest that BDNF and NT-3 increase the number of nerve terminals and synaptic contacts. Thus, the neurotrophins not only enhanced the expression of synaptic vesicle proteins, but also induced morphological differentiation of presynaptic terminals.

\section{Discussion}

Xenopus neuromuscular synapses undergo a maturation process in culture similar to the one in vivo (Kullberg et al., 1977; Nakajima et al., 1980). A number of electrophysiological parameters can be used to monitor the progress of synaptic maturation. There is a gradual increase of the frequency and amplitude as well as a decrease in the rise time of SSCs (Evers et al., 1989). The transition from a skew- to a bell-shaped SSC amplitude distribution usually occurs around 4-5 d in culture (Kidokoro, 1984; Lu et al., 1992). The amplitude of ESCs becomes much larger and more consistent (Evers et al., 1989). A major finding of the present study is that N'I-3 and BDNF accelerated the progression of synaptic maturation, as measured by the above clectrophysiological parametcrs. The increasc in the frequency of SSCs and the amplitudes of ESCs upon acute application of BDNF and NT-3 to the same culture system has been recently reported (Lohof et al., 1993). The chronic effects of the neuro- 

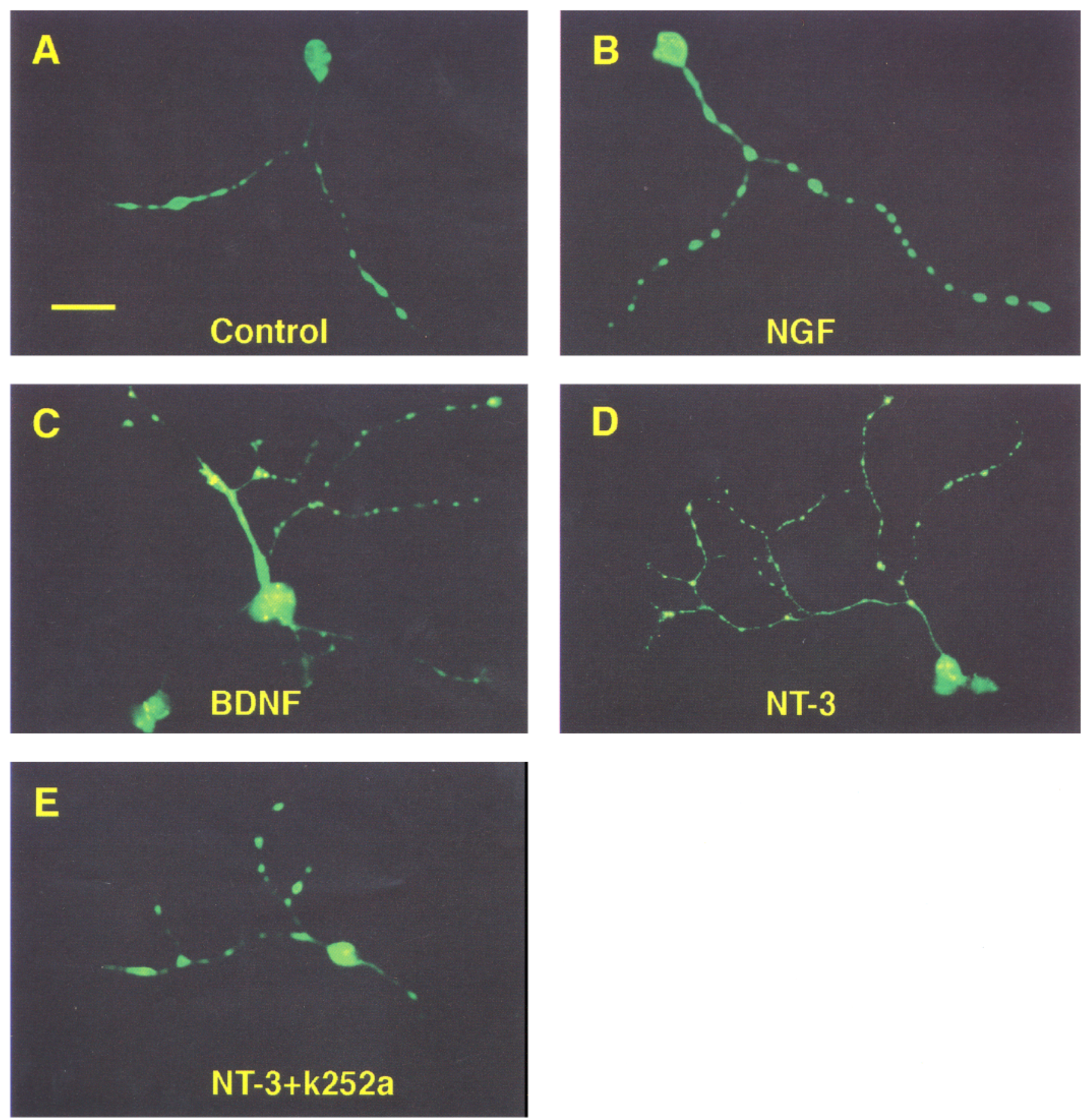

Figure 5. Effects of NT-3 on synaptophysin expression. Dissociated, 3-d-old Xenopus nerve-muscle cultures were fixed and processed for standard indirect immunofluorescence histochemistry, using an anti-synaptophysin antibody. Note the increase of synaptophysin staining as well as the number of varicosities in BDNF- and NT-3-treated neurons. Scale bar, $20 \mu \mathrm{m}$.

trophins, however, showed several distinctive features, when compared with acute effects of the factors observed previously. For example, long-term BDNF or NT-3 treatment resulted in an increase in the mean amplitude and a decrease in the rise time of SSCs (Table 1). A morc dramatic change was the earlier appearance of a bell-shaped distribution of SSC amplitudes (Figs. $1,2)$. No such changes were seen after acute exposure of neurotrophins (Lohof et al., 1993). Moreover, the acute effects of NT-3 are transient, depending on a continuous presence of the factors. In contrast, chronic exposure of NT-3 elicited a profound change in synaptic efficacy that persists even after withdrawal of the neurotrophin. Long-term neurotrophin treatment also enhanced the levels of presynaptic vesicle proteins (Fig. 7). Finally, BDNF and NT-3 induced a morphological change of presynaptic neurons: the number of varicosities increased significantly (Fig. 8). Thus, the acute application of neurotrophins induces transient change in the probability of transmitter release, possibly through phosphorylation. In contrast, the chronic ef- 

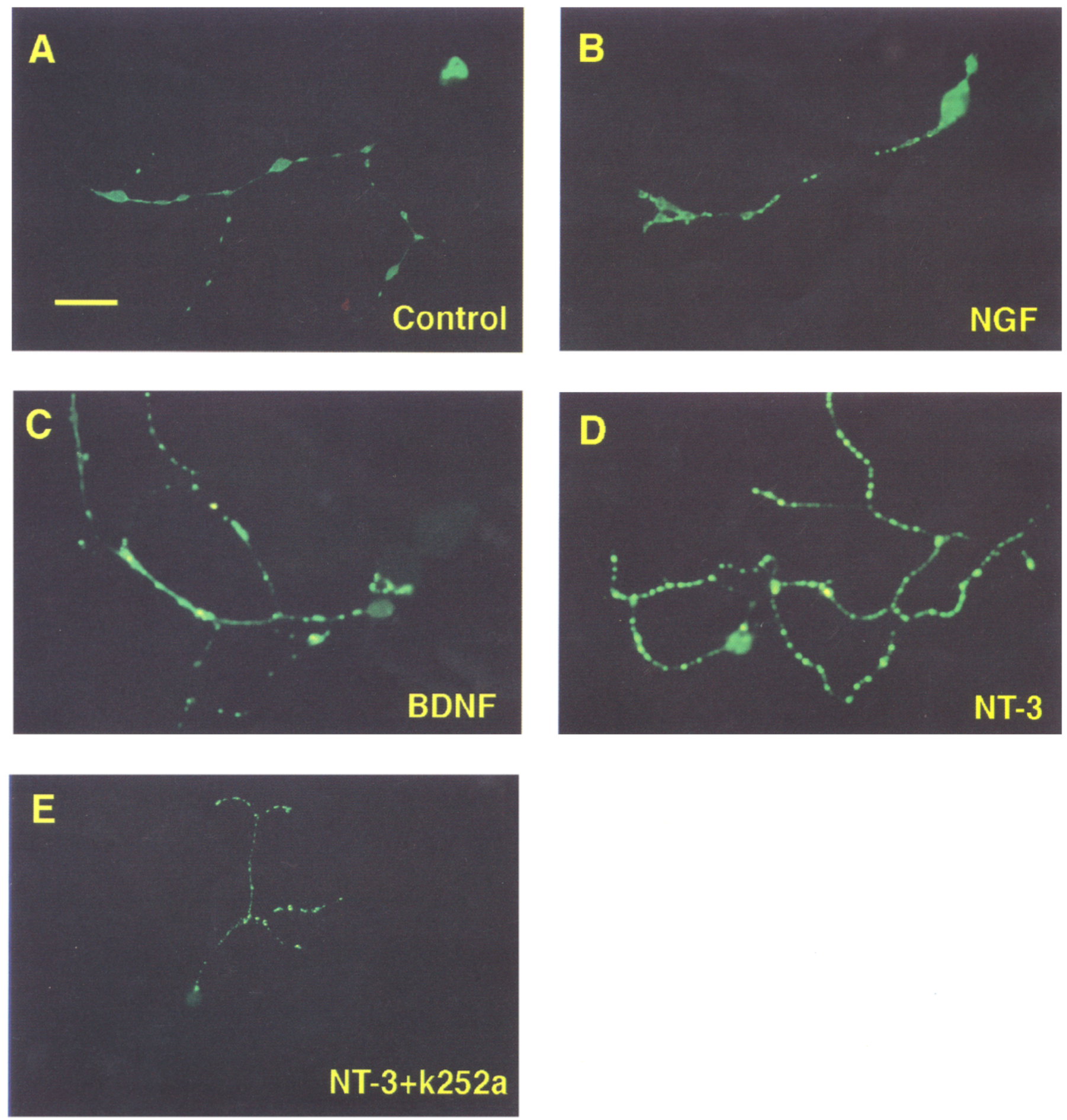

Figure 6. Effects of NT-3 on the expression of synapsin I. Three-day-old nerve-muscle cultures were stained with an antibody against Xenopus synapsin I. Note the increase of synapsin I staining in BDNF- and NT-3-treated neurons. Also notice that the number of varicosities in cultures treated with BDNF and NT-3 were markedly increased. Scale bar, $20 \mu \mathrm{m}$.

fects of neurotrophins may involve gene regulation that ultimately leads to long-term structural and functional maturation of the synapses.

The higher frequency and amplitude of SSCs observed at synapses after neurotrophin treatment could result from pre- and/or postsynaptic changes. A higher SSC amplitude could be due to a larger amount of $\mathrm{ACh}$ per quantal package in the presynaptic terminals or to an increase in postsynaptic ACh sensitivity. An increase in SSC frequency usually indicates a presynaptic effect: either more releasable ACh packages are available or the probability of release is increased. However, a potentiation of postsynaptic ACh responsiveness could also elevate synaptic currents normally hidden within the recording noise and thus increase the apparent SSC frequency. Our cell manipulation 


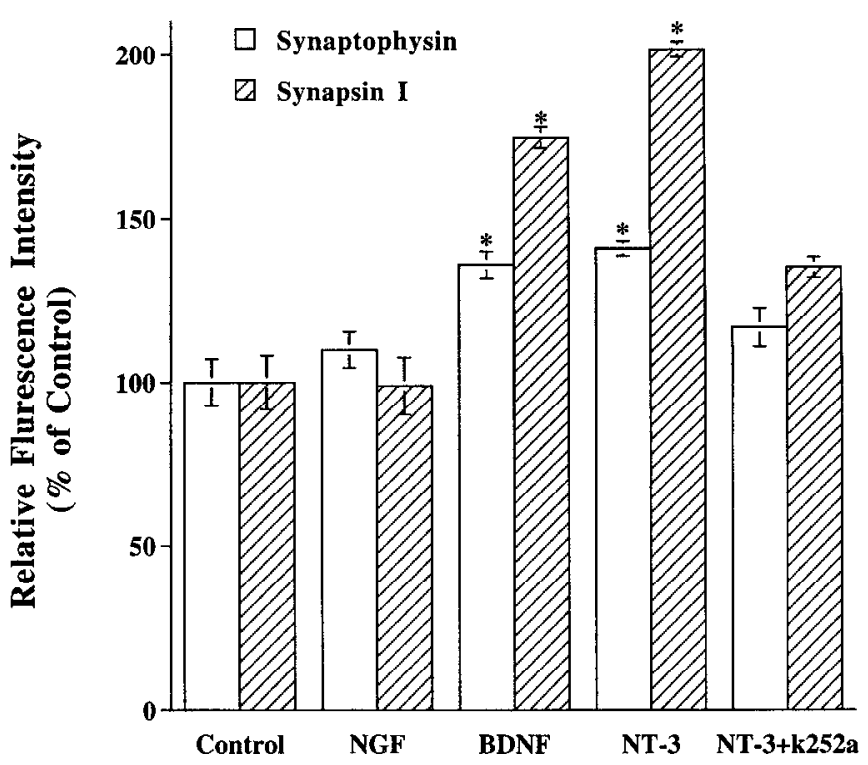

Figure 7. Quantitation of neurotrophin effects on the staining of synapsin I and synaptophysin. Images of spinal neurons in $3 \mathrm{~d}$ cultures grown in different conditions as indicated were analyzed by the Adobe PHOTOSHOP program. Data are expressed as percentage of control (mean \pm SEM). Each column represents relative staining intensity on varicosities averaged from five to eight spinal neurons. ${ }^{*}$ Significantly different $(t$ test, $p<0.001$ )

experiments indicated that ACh pulses from NT-3-treated nerve terminals were obviously bigger and more frequent. In addition, NT-3 induced the expression of synaptic vesicle proteins. Interestingly, NT-3 treatment did not elicit significant changes of decay time, although the reduction of SSC decay time occurs during normal development of neuromuscular synapses. This offers additional evidence that NT-3 acts on presynaptic nerve terminal without affecting postsynaptic ACh receptors. All these observations are in favor of a presynaptic action of neurotrophins.

Exactly how BDNF and NT-3 enhance synaptic efficacy is unknown. A previous study showed that the neurotrophins induced choline acetyltransferase (CAT) activity in motor terminals (Wong et al., 1993). However, enhancement of ACh synthesis can only partially explain the changes in synaptic physiology. Assuming that BDNF and NT-3 increase ACh levels uniformly in all vesicles, one could predict a general increase in the frequency and amplitude of SSCs. This would not Icad to the appearance of a bell-shaped amplitude distribution nor a change in the rise time. A more likely mechanism is that BDNF and NT-3 enhance the loading of ACh to unfilled synaptic vesicles. This will lead to uniformed vesicles and, thereby, a bellshaped distribution of SSC amplitude. Neurotrophic regulation of synapsin I expression at neuromuscular synapses may contribute to mechanisms underlying the enhancement of synaptic efficacy. Exogenous synapsin I loaded into Xenopus spinal neurons accelerates structural and functional maturation of neuromuscular synapses (Lu et al., 1992; Valtorta et al., 1995). In addition, when endogenous synapsin I was induced to express earlier neuromuscular synapses matured much earlier ( $\mathrm{Lu}$ et al., unpublished observations). Since synapsin I can cause aggregation of synaptic vesicles (Benfenati et al., 1994), it is conceivable that the interaction of synapsin I with synaptic vesicles and cytoskeletons may help the assembly of mature transmitter release machinery, including the development of active zones

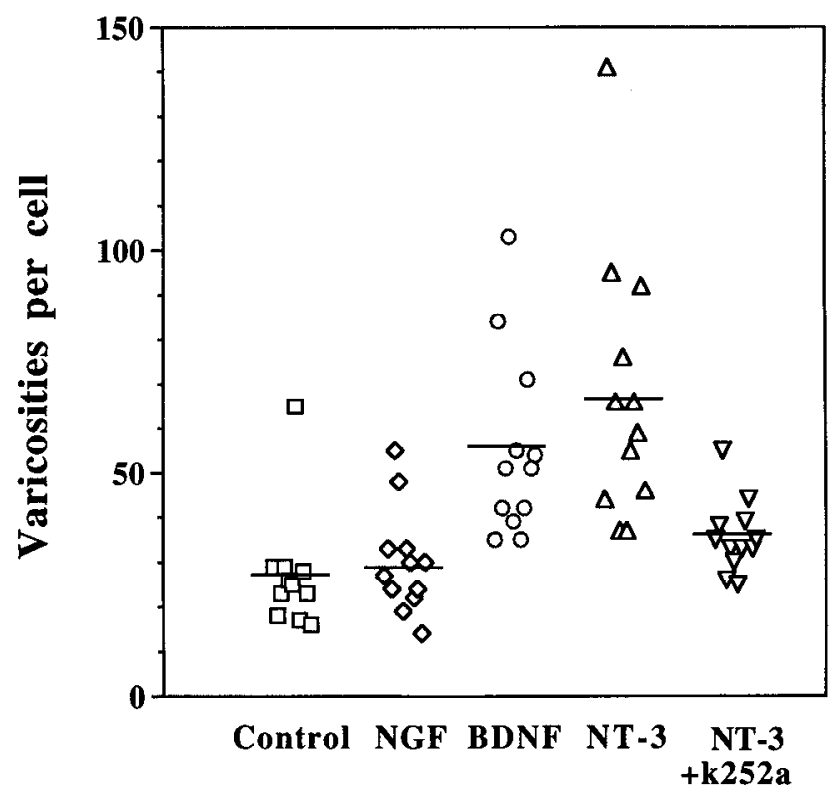

Figure 8. Scatted plot of neurotrophin effects on the number of varicosities per neuron. Each data point represents number of varicosities of a single spinal neuron. The horizontal lines indicate the mean values of control and neurotrophin-treated groups. The BDNF and NT-3 groups are significantly different from the control group ( $t$ test, $p<$ 0.001 ). The NT $-3+\mathrm{k} 252 \mathrm{a}$ group is not significantly different from the control group.

and loading of $\mathrm{ACl}$ to synaptic vesicles. However, knockout mice lacking the synapsin I gene manifest no significant changes in their synaptic architecture (Rosahl et al., 1994). It is unclear whether synapses in the mutant mice undergo normal maturation process during development. In the present study, we have shown that NT-3-enhanced synapsin I staining at these terminals (Figs. 6,7). It is tempting to think that neurotrophins elicit their effects by inducing synapsin I expression. Whether neurotrophin-induced synaptic maturation is, indeed, mediated by synapsin I requires further investigation.

BDNF and NT-3 also enhance the survival and neurite extension of spinal neurons in Xenopus nerve muscle cultures (Wang and Lu, unpublished observation; M.-m. Poo, personal communication). The neurotrophins have also been shown to upregulate the CAT activity in embryonic motor neurons (Wong et al., 1993). These findings raised the concern whether the potentiation of synaptic maturation obscrved in the present study is merely a natural consequence of motor neuron differentiation induced by the neurotrophins. However, several lines of evidence suggest that the effects of the neurotrophins on synapse maturation are distinguishable from those on general neuronal differentiation. First, BDNF, although it enhances survival and neurite extension better than NT-3, was less effective in promoting synaptic maturation (Table 1). Second, an overall increase in the CAT activity in spinal neurons cannot explain the appearance of a bell-shaped amplitude distribution of SSCs. Furthermore, longer and more branched neurites do not automatically confer more mature synapses with reliable synaptic efficacy. In fact, we detected virtually no SSCs on neurites between varicosities in neurons grown in NT-3 (Lu et al., unpublished observations). It appears that transmitter release capacity became more restricted to the varicosities or nerve terminals after neurotrophin treatment. Nevertheless, it is difficult to fully establish that BDNF and NT-3 exert distinct effects on synaptic matura- 
tion, independent of their effects in promoting neuronal survival and neurite outgrowth.

\section{References}

Alder J, Lu B, Valtorta F, Greengard P, Poo MM (1992) Calciumdependent transmitter secretion reconstituted in Xenopus oocytes: requirement for synaptophysin. Science 257:657-661.

Barbacid M (1993) Nerve growth factor: a tale of two receptors. Oncogene 8:2033-2042.

Barde Y (1989) Trophic factors and neuronal survival. Neuron 2:1525-1534.

Benfenati $F$, Valtorta F, Rossi MC, Onofri F, Sihra T, Greengard P (1994) Interaction of synapsin I with phospholipids: possible role in synaptic vesicle clustering and in the maintenance of bilayer structures. J Cell Biol 123:1845-1855.

Berg MM, Sternberg DW, Parada LF, Chao MV (1992) K-252a inhibits nerve growth factor-induced trk proto-oncogene tyrosine phosphorylation and kinase activity. J Biol Chem 267:13-16.

Buchanan J, Sun Y, Poo MM (1989) Studies of nerve-muscle interactions in cell culture: fine structure of early functional contacts. J Neurosci 9:1540-1554.

Chao MV (1992) Neurotrophin receptors: a window into neuronal differentiation. Neuron 9:583-593.

Ernfors P, Lee K, Jaenisch R (1994a) Mice lacking brain-derived neurotrophic factor develop with sensory deficits. Nature 368:147-150.

Ernfors P, Lee K, Kucera J, Jaenisch R (1994b) Lack of neurotrophin-3 leads to deficiencies in the peripheral nervous system and loss of limb proprioceptive afferents. Cell 77:503-512.

Evers J, Laser M, Sun YA, Xie ZP, Poo MM (1989) Studies of nervemuscle interaction in Xenopus cell culture: analysis of early synaptic currents. J Neurosci 9:1523-1539.

Farinas I, Jones KR, Backus C, Wang S, Reichardt LF (1994) Severe sensory and sympathetic deficits in mice lacking neurotrophin-3. Nature 369:658-661

Greengard P, Valtorta F, Czernik AJ, Benfenati F (1993) Synaptic vesicle phosphoproteins and regulation of synaptic function. Science 259:780 785

Hall ZW, Sanes JR (1993) Synaptic structure and development: the neuromuscular junction. Cell/Neuron Rev [Suppl] 10:99-122.

Hanill OP, Marty A, Neher E, Sakmann B, Sigworth FJ (1981) In1proved patch clamp techniques for high-resolution current recording from cell and cell-free membrane patches. Pfluegers Arch 391:85100 .

Henderson CE, Camu W, Mettling C, Gouin A, Poulsen K, Karihaloo M, Rullamas J, Evens T, MaMahon SB, Armanini MP, Berkemeier L, Phillips HS, Rosenthal A (1993) Neurotrophins promote motor neuron survival and are present in embryonic limb bud. Nature 363: $268-270$.

Ip NY, Li Y, Yancopoulos G, Lindsay R (1993) Cultured hippocampal neurons show responses to BDNF, NT-3, and NT-4, but not NGF $J$ Neurosci 13:3391-3105.

Jones KR, Farinas I, Backus C, Reichardt LF (1994) Targeted disruption of the BDNF gene perturbs brain and sensory neuron development but not motor neuron development. Cell 76:989-999.

Kelly AM, Zachs SI (1969) The fine structure of motor end-plate morphogenesis. J Cell Biol 42:154-169.

Kidokoro Y (1984) Two types of miniature endplate potentials in Xenopus nerve muscle cultures. Neurosci Res 1:157-170.

Kidokoro Y, Anderson MJ, Gruner R (1980) Changes in synaptic potential properties during acetylcholine receptor accumulation and neurospecific interactions in Xenopus nerve-muscle cell culture. Dev Biol 78:464-483.

Klein R, Smeyne RJ, Wurst W, Long LK, Auerbach BA, Joyner AL, Barbacid M (1993) Targeted disruption of the trkB neurotrophin receptor gene results in nervous system lesions and neonatal death. Cell $75: 113-122$.

Koliatsos VE, Clatterbuck RE, Winslow JW, Cayouette MH, Price DL
(1993) Evidence that brain-derived neurotrophic factor is a trophic factor for motor neurons in vivo. Neuron 10:359-367.

Kullberg RW, Lentz TL, Cohen MW (1977) Development of myotomal neuromuscular junction in Xenopus laevis: an electrophysiological and fine structural study. Dev Biol 60:101-129.

Lohof AM, Ip NY, Poo MM (1993) Potentiation of developing neuromuscular synapses by the neurotrophins NT-3 and BDNF. Nature $363: 350-353$.

Lu B, Greengard P, Poo MM (1992) Exogenous synapsin I promotes functional maturation of developing neuromuscular synapses. Neuron $8: 521-529$.

Nakajima Y, Kidokoro Y, Klier FG (1980) The development of functional neuromuscular junction in vitro: an ultrastructural and physiological study. Dev Biol 77:52-72.

Nieuwkoop P, Faber P (1967) Normal table of Xenopus laevis. Amsterdam: North Holland.

Nye SH, Squinto SP, Glass DJ, Stitt TN, Hantzopoulos P, Macchi MJ, Lindsay NS, Ip NY, Yancopoulos GD (1992) K-252a and staurosporine selectively block autophosphorylation of neurotrophin receptors and neurotrophin-mediated responses. Mol Biol Cell 3:677-686.

Oppenheim RW (1991) Cell death during development of nervous system. Annu Rev Neurosci 14:453-501.

Oppenheim RW, Yin Q, Prevette D, Yan Q (1992) Brain-derived neurotrophic factor rescues developing avian motor neurons from cell death. Nature 360:755-757

Purves D, Lichtman JW (1985) Principle of neural development. Sunderland: Sinauer.

Rosahl TW, Geppert M, Spillane D, Herz J, Hammer RE, Malenka RC, Sudhof TC (1994) Short-term synaptic plasticity is altered in mice lacking synapsin I. Cell 75:661-670.

Schecterson LC, Bothwell M (1992) Novel Roles for neurotrophins are suggested by BDNF and NT-3 mRNA expression in developing neurons. Neuron 9:449-463.

Sendtner M, Holtmann B, Kolbeck R, Thoenen H, Barde Y (1992) Brain-derived neurotrophic factor prevents the death of motoneurons in newborn rats after nerve section. Nature 360:757-759.

Sudhof TC, Jahn R (1991) Proteins of synaptic vesicles involved in exocytosis and membrane recycling. Neuron 6:665 677 .

Tabti N, Poo MMM (1991) Culturing spinal neurons and muscle cells from Xenopus embryos. In: Culture of nerve cells (Goslin GBK, ed), pp 137-153. Buston: MIT Press.

Takahashi T, Nakajima Y, Hirosawa K, Nakajima S, Onodera K (1987) Structure and physiology of developing neuromuscular synapses in culture. J Neurosci 7:473-481.

Tapley P, Lamball F, Barbacid M (1992) K252a is a selective inhibitor of the tyrosine protein kinase activity of the trk family of oncogenes and neurotrophin receptors. Oncogene 7:371-381.

Thoenen H (1991) The changing scene of neurotrophic factors. Trends Neurosci 14:165-170.

Valtorta F, Villa A, Jahn R, DeCamilli P, Greengard P (1988) Localization of synapsin $I$ at the frog neuromuscular junction. Neuroscience 21:593-603.

Valtorta F, Lezzi N, Benfenati F, Lu B, Poo MM, Greengard P (1995) Accelerated structural maturation induced by synapsin I at developing neuromuscular synapses. Eur $\mathbf{J}$ Neurosci, in press.

Wong V, Arriaga R, Ip NY, Lindsay RM (1993) The neurotrophins BDNF, NT-3 and NT-4/5, but not NGF, upregulate the cholinergic phenotype of developing motor neurons. Eur J Neurosci 5:466-474.

Xie ZP, Poo MMM (1986) Initial events in the formation of neuromuscular synapse: rapid induction of acetylcholine release from embryonic neuron. Proc Natl Acad Sci USA 83:7069-7073.

Yan Q, Elliott J, Snider WD (1992) Brain-derived neurotrophic factor rescues spinal motor neurons from axotomy-induced cell death. Nature 360:753-755.

Yin Q, Oppenheim RW (1992) Modification of motor neuron development following transplantation of thoracic spinal cord to the lumbar region in the chick embryo: evidence for target-derived signals that regulate differentiation. J Neurobiol 23:376-395. 